\title{
Development of SPR and fluorescence synchronous detection system
}

\author{
L.L. Zhang, X. Chen, D.F. Cui, Y.T. Li, Y. F. Ren, C.F. Xu \\ State key laboratory of transducer technology, Institute of Electronics, Chinese Academy of Sciences, \\ Beijing 100190, China \\ E-mail: Ilzhang@mail.ie.ac.cn
}

\begin{abstract}
:
Surface plasmon resonance (SPR) biosensors was an extremely sensitive optical technique to detect the changes in refractive index occurring at the sensor interface. SPR biosensors and fluorescence analysis were two kinds effective methods for real-time detection. The combination of these technology would improve the detection sensitivity of fluorescence quantitative and the specificity of SPR detection. We designed and developed a SPR and fluorescence synchronous detection system. SPR module was based on two kinds of modulation methods and fluorescence module could switch among 4 kinds of wavelength. SPR peak curves of fluorescent microspheres was collected by SPR angular scanning method.
\end{abstract}

Key words: Surface plasmon resonance (SPR), Fluorescence, Combination, Sensitivity, Biosensor

\section{Introduction}

As a label-free technology, SPR relies on the change of molecular weight of the detected substances. It is difficult to detect small molecules, and it is obtained a complex information of the sample to be measured by detecting the refractive index changes. Fluorescence detection is a specific detection method that has been widely used in the field of modern biochemical research and disease diagnosis. However, the fluorescence detection method is not easy to realize quantitative. Therefore, the combination of SPR method and fluorescence technology can achieve complementary advantages.

In 1991, Attridge et al. first reported the detection of hCG by the fluorescence analysis method based on SPR. In 2002, Gast monitored a variety of components of the molecular adsorption process using the simultaneous SPR and fluorescence information. In 2014, the Iwata team developed a surface plasma enhanced fluorescence spectrum system for POCT diagnosis. But this POCT diagnostic device is based on fixed angle detection mode, and the detection range of refractive index is limited.

In this paper, a SPR and fluorescence synchronous detection system was developed. The system was based on two kinds of modulation methods which included angle scanning and intensity detection. In the mean while the system can also detect the fluorescence signal by switching light wavelength among DAPI, FITC, TX RED and CY5.

\section{Experiments and Results}

The SPR sensor was based on the prism coupling mode of the Kretschmann structure. As shown in Figure 1, the proposed SPR sensor consisted a red laser light source (635nm peak wavelength), a prism with an equilateral triangle shape $(n=1.72$; Beijing Glass Factory, China), SPR sensor chip, and a CCD camera (Basler, A102f). The laser, the polarizing filter and the lens system were installed on a rotating arm. The CCD was mounted on another rotating arm and both rotating arms were controlled by a stepper motor. The fluorescence detector located on top of the SPR sensor. It contained light sauce, filters, beam splitter, objective lens, imaging lens and CCD. The fluorescence system had two working modes: common fluorescence and enhanced fluorescence. In common fluorescence mode, mercury lamp generated incident light, passed through the filter and was reflected into the objective lens by the beam splitter. In the enhanced fluorescence mode, excitation light was generated by the field of the evanescent wave. The fluorescence emitted by 
the fluorescent substance would be conducted to the CCD. Figure 2 shows the configuration design of the system.

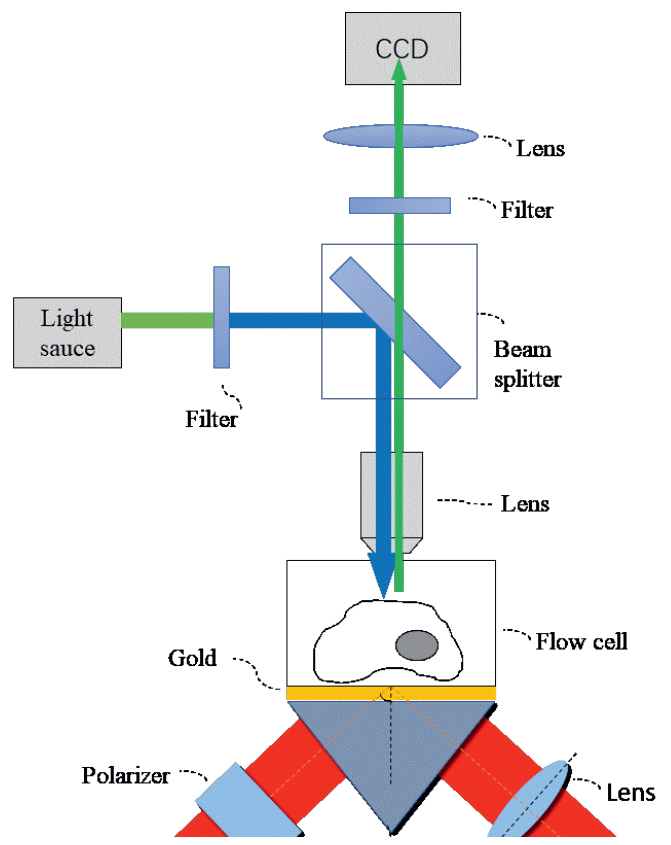

Fig.1. SPR and fluorescence synchronous detection system optical structure

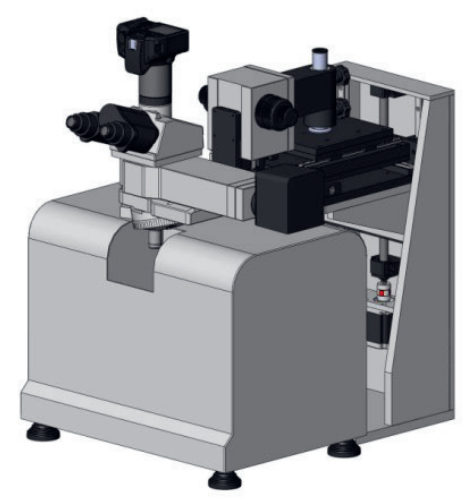

Fig.2. The configuration design of SPR and fluorescence synchronous detection system

During the SPR experiment, SPR gold sensor chip was placed on top of the prism adding the index matching oil between the chip and the prism. A scan of the incident light angle was conducted in Milli-Q water to locate the absorption peak and the corresponding couple angle. Then fluorescent microspheres were added on the surface of the SPR chip, incident light angle was changed by a motor and mechanical device. Fig. 3 showed the experiment results of the SPR peak curves of fluorescent microspheres by angular scanning from 90000 to 130000 steps. We can fix the incident angle at 110000 steps and monitor the SPR reflected light intensity and take the fluorescent picture.

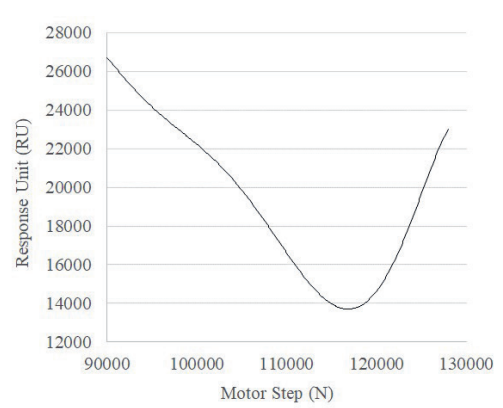

Fig.3. SPR peak curves of fluorescent microspheres by angular scanning

\section{Summary}

SPR biosensors and fluorescence analysis were combined and a real-time system were developed. SPR module was based on angle scanning and intensity modulation methods. The fluorescence module could switch among 4 kinds of wavelength. SPR peak curves of fluorescent microspheres was collected by SPR angular scanning method. The system would be used in cell analysis and molecule diagnosis in the future.

\section{Acknowledgements}

The authors greatly acknowledge the financial support from the National Natural Science Foundation of China (Grant Nos. 61571420, 31571007 and 61201079).

\section{References}

[1] Homola J., Surface Plasmon Resonance Sensors for Detection of Chemical and Biological Species. Chem. Rev., 2008, 108 (2): $462-493$.

[2] Liebermann T., Knoll W., Surface plasmon field-enhanced fluorescence spectroscopy, Colloids Surf. A, 2000, 171: 115-130.

[3] Attridge J. W., Daniels P. B., Deacon J. K., Robinson G. A., Davidson G. P., Sensitivity enhancement of optical immunosensors by the use of a surface plasmon resonance fluoroimmunoassay, Biosensors and Bioelectronics, 1991, 6( 3): 201-214.

[4] Roy S., Kim J., James T. Kellis J., Poulose A., Robertson C. R., Gast A. P., Surface plasmon resonance/surface plasmon enhanced fluorescence: an optical technique for the detection of multicomponent macromolecular adsorption at the solid/liquid interface, Langmuir, 2002,18( 16): 6319-6323.

[5] Toda M., Arima Y., Takiguchi H., Iwata H. Surface plasmon field-enhanced fluorescence spectroscopy apparatus with a convergent optical system for point-of-care testing, Analytical biochemistry, 2014, 467: 47-53. 